\title{
Comorbidities and Quality of Life in Women Undergoing First Surgery for Endometriosis: Differences Between Chinese and Italian Population
}

\author{
Huixi Chen ${ }^{1,2,3} \cdot$ Silvia Vannuccini ${ }^{1,4}(1) \cdot$ Tommaso Capezzuoli $^{1} \cdot$ Marcello Ceccaroni $^{5} \cdot$ Liu Mubiao $^{6} \cdot$ Huang Shuting $^{6}$. \\ Yanting $\mathrm{Wu}^{2,3} \cdot$ Hefeng Huang ${ }^{2,3} \cdot$ Felice Petraglia ${ }^{1}$ (10)
}

Received: 12 July 2020 / Accepted: 2 February 2021 / Published online: 9 March 2021

(C) The Author(s) 2021

\begin{abstract}
An observational cross-sectional study was conducted in a group $(n=371)$ of fertile age women with endometriosis, by administering a structured questionnaire, in order to evaluate the incidence of gynecological and systemic comorbidities and the impact on quality of life (QoL) in two different groups of Italian and Chinese patients affected by endometriosis. Chinese $(n=$ $175)$ and Italian $(n=196)$ women were compared regarding systemic (inflammatory, autoimmune, and mental) and gynecological comorbidities, pain symptoms, and QoL, by using the Short Form 12 (SF-12). Italian patients resulted younger at the diagnosis and suffered more frequently from severe pain than Chinese ones. Deep infiltrating endometriosis (DIE) and mixed phenotypes were more frequent in Italian patients, whereas ovarian (OMA) and superficial endometriosis (SUP) were more common in the Chinese. The Italian group showed more systemic comorbidities, and those disorder were already present before the diagnosis of endometriosis. Furthermore, the Italian group showed lower SF-12 physical and mental scores, suggesting a worse health-related QoL in Italian endometriotic patients. A number of differences has been observed between Italian and Chinese women with endometriosis in terms of comorbidities and QoL, which may be related to the ethnicity, the different health system organization and the social and cultural background.
\end{abstract}

Keywords Autoimmune disorders · Comorbidities · Endometriosis · Ethnicity $\cdot$ Inflammatory diseases $\cdot$ Metabolic disorders . Psychiatric diseases · Quality of life

Huixi Chen and Silvia Vannuccini are joint first authors.

Felice Petraglia

felice.petraglia@unifi.it

1 Obstetrics and Gynecology, Department of Experimental and Clinical Biomedical Sciences, Careggi University Hospital, University of Florence, Largo Brambilla, 3, 50134 Florence, Italy

2 International Peace Maternity and Child Health Hospital, School of Medicine, Shanghai Jiao Tong University, Shanghai, China

3 Shanghai Key Laboratory of Embryo Original Disease, Shanghai, China

4 Department of Molecular and Developmental Medicine, University of Siena, Siena, Italy

5 Gynecology and Obstetrics, Gynecologic Oncology, Minimally-Invasive Pelvic Surgery, International School of Surgical Anatomy, IRCCS Sacro Cuore Don Calabria Hospital, Negrar di Valpolicella, Verona, Italy

6 Guangdong Provincial People's Hospital, Guangdong Academy of Medical Sciences, Guangdong, China

\section{Introduction}

Endometriosis is a benign gynecological disease characterized by an estrogen-dependent inflammatory process with ectopic localization of endometrial cells, affecting approximately $10 \%$ of reproductive aged women. Debilitating pain-related symptoms (dysmenorrhea, dyspareunia, dysuria, and dyschezia) and infertility are commonly observed in affected women. A negative association exists between endometriosis-associated pain and daily activities, self-care, and productivity at work up to job loss, and these may cause a low quality of life [1]. Medical or surgical treatment of endometriosis aim to achieve a complete and durable symptom relief, with good physical and mental health [2]; however, the disease is chronic and recurrent [3].

Furthermore, endometriosis is associated with gynecological [4] and systemic comorbidities, including immune (asthma, rheumatoid arthritis, psoriasis, and multiple sclerosis), inflammatory (bowel inflammatory disease and Crohn's disease), and psychiatric disorders (depression and anxiety ) [5-7]. 
The majority of epidemiological data on endometriosis comes from reports on Caucasian women [8]. However, also Asian women are likely to be diagnosed with endometriosis, whereas African-American women are less frequently affected [9-11]. Few evidences are available on the Chinese population [12], but there is a high number of patients with endometriosis in China because of the large population base. Moreover, information on women with endometriosis differentiated by ethnicity are quite limited. The present study aims to evaluate the difference between Chinese and Italian population with endometriosis in terms of symptoms, comorbidities, and Quality of Life (QoL).

\section{Materials and Methods}

An observational cross-sectional multicenter study was conducted in a group $(n=371)$ of fertile age women $(25-45$ years old) with endometriosis, recruited in four different hospitals, all third level centers for endometriosis treatment (Florence and Negrar di Valpolicella, Verona, Italy; Shanghai and Guangdong, China). They were divided into two agematched groups based on the ethnicity: (a) Chinese population ( $n=175)$ and (b) Italian population $(n=196)$. We included only women with histological diagnosis of endometriosis. Data were collected within 2 years after a single surgery for endometriosis through a structured questionnaire, during a clinical follow-up visit. Women with previous or actual pregnancy were excluded. Also women with multiple surgical interventions for endometriosis were not included in the study. A database was built collecting for each case all the following information:

a) demographic characteristics (age, body mass index [BMI], and age at menarche);

b) endometriosis data: age at the diagnosis of endometriosis, phenotype of endometriosis at surgery (ovarian endometriosis [OMA], deep infiltrating endometriosis [DIE], superficial endometriosis [SUP], and mixed phenotype), if hormonal medical treatment before and after surgery was administered, and current symptoms (dysmenorrhea, dyspareunia, non-menstrual pelvic pain, and urinary pain), measured as absent, mild, moderate, and severe, according to the visual analog scale (VAS). In fact, women graded their perception of each type of pain on a $10-\mathrm{cm}$ line from 0 (no pain) to 10 (unbearable pain); a mean VAS score of 7 or higher was considered severe;

c) gynecological (uterine fibroids, adenomyosis, and polycystic ovarian syndrome) and systemic comorbidities, including autoimmune (thyroiditis, rheumatoid arthritis, psoriasis, pemphigus, multiple sclerosis, and myasthenia gravis), metabolic (obesity, hypertension, and hypercholesterolemia), inflammatory (allergic rhinitis, allergic asthma, irritable bowel syndrome, and inflammatory bowel diseases), and mental health diseases (depression, anxiety and panic disorder, eating disorders); it has been also asked whether the disorder appears before or after the diagnosis of endometriosis;

d) evaluation of health-related Quality of Life (QoL) by using the Short Form-12 (SF-12) [13].

The data were validated through review of medical records of all participants to confirm what was reported by patients, especially for systemic comorbidities. The study was approved by the locals Institutional Review Boards, and all participants provided written informed consent to be included in the series.

\section{Statistical Analysis}

Statistical analysis was performed using IBM SPSS Statistics software, version 22 (IBM Corporation, Armonk, NY, USA). Statistically significant differences between groups were determined using Student's $t$-test for continuous variables and chi-square test or Fisher's exact test for categorical variables. A $p$ value $<0.05$ was considered statistically significant. The sample size was estimated to detect differences of at least 0.5 standard deviations in quantitative variables or $20 \%$ in the frequency of categorical variables between groups with $80 \%$ statistical power and $95 \%$ confidence level. In order to achieve a statistical power of $95 \%$, we needed at least 111 patients with a significance level of 0.05 and an effect size of 0.3 .

\section{Results}

The group of Italian women resulted younger than the Chinese at diagnosis of endometriosis $(p<0.0001)$. Chinese women were more frequently diagnosed with OMA ( $84.0 \%$ vs $46.7 \%$ ), whereas Italians had significantly more DIE (4.1\% vs $1.8 \%)$ and mixed phenotypes ( $40 \%$ vs $5.6 \%)(p<0.0001)$ (Table 1). Furthermore, the group of Italian women underwent more frequently to medical hormonal treatment before surgery (21\% vs $43.3 \%, p<$ $0.0001)$. On the contrary, no differences were found in terms of indications for surgery, which was mainly represented by pain symptoms (Table 1).

Regarding gynecological comorbidities, PCOS $(7.9 \%$ vs $5.3 \%)$ and uterine fibroids (9.3\% vs $3.2 \%$ ) were more common in the Chinese than in Italian patients, while adenomyosis was more common in Italians (27.9\% vs $9.9 \%)$. Most of the systemic comorbidities observed were more common in Italian women than in the Chinese, i.e., autoimmune diseases (27.5\% vs $4.6 \%, p<0.0001)$, metabolic/endocrine diseases ( $14.8 \%$ vs $8.7 \%, p=0.007)$, inflammatory diseases $(44.5 \%$ vs 
Table 1 Clinical characteristics of the two populations

\begin{tabular}{|c|c|c|c|}
\hline & Chinese $(n=175)$ & Italian $(n=196)$ & $p$ value \\
\hline Age (years) & $36.5 \pm 6.7$ & $36.3 \pm 6.4$ & 0.769 \\
\hline BMI & $21.4 \pm 3.1$ & $22.2 \pm 3.3$ & 0.021 \\
\hline Menarche (years) & $13.5 \pm 1.6$ & $12.3 \pm 1.3$ & $<0.0001$ \\
\hline Age at first diagnosis (years) & $32.2 \pm 5.9$ & $27.3 \pm 5.6$ & $<0.0001$ \\
\hline Medical treatment before first surgery & $34(21.0 \%)$ & $85(43.4 \%)$ & $<0.0001$ \\
\hline \multicolumn{4}{|l|}{ Indication for first surgery } \\
\hline Pain & $92(54.8 \%)$ & $104(53.1 \%)$ & \multirow[t]{4}{*}{0.025} \\
\hline Pain and infertility & $7(4.2 \%)$ & $24(12.2 \%)$ & \\
\hline Infertility & $8(4.8 \%)$ & $4(2 \%)$ & \\
\hline Imaging & $61(36.3 \%)$ & $64(32.7 \%)$ & \\
\hline \multicolumn{4}{|l|}{ Endometriosis phenotype at surgery } \\
\hline OMA & $137(84.0 \%)$ & $91(46.7 \%)$ & \multirow[t]{7}{*}{$<0.0001$} \\
\hline DIE & $3(1.8 \%)$ & $8(4.1 \%)$ & \\
\hline SUP & $14(8.6 \%)$ & $14(7.2 \%)$ & \\
\hline $\mathrm{OMA}+\mathrm{DIE}$ & $4(2.5 \%)$ & $26(13.3 \%)$ & \\
\hline $\mathrm{OMA}+\mathrm{DIE}+\mathrm{SUP}$ & $0(0.0 \%)$ & $25(12.8 \%)$ & \\
\hline $\mathrm{OMA}+\mathrm{SUP}$ & $5(3.1 \%)$ & $24(12.3 \%)$ & \\
\hline DIE+SUP & $0(0.0 \%)$ & $7(3.6 \%)$ & \\
\hline \multicolumn{4}{|l|}{ Dysmenorrhea } \\
\hline No & $52(36.6 \%)$ & $60(31.7 \%)$ & \multirow[t]{4}{*}{$<0.0001$} \\
\hline Mild & $56(39.4 \%)$ & $34(18.0 \%)$ & \\
\hline Moderate & $14(9.9 \%)$ & $52(27.5 \%)$ & \\
\hline Severe & $20(14.1 \%)$ & $43(22.8 \%)$ & \\
\hline \multicolumn{4}{|l|}{ Dyspareunia } \\
\hline No & $94(76.4 \%)$ & $57(29.7 \%)$ & \multirow[t]{4}{*}{$<0.0001$} \\
\hline Mild & $24(19.5 \%)$ & $43(22.4 \%)$ & \\
\hline Moderate & $3(2.4 \%)$ & $66(34.4 \%)$ & \\
\hline Severe & $2(1.6 \%)$ & $26(13.5 \%)$ & \\
\hline \multicolumn{4}{|l|}{ Non menstrual pelvic pain } \\
\hline No & $92(66.2 \%)$ & $57(29.5 \%)$ & \multirow[t]{4}{*}{$<0.0001$} \\
\hline Mild & $35(25.2 \%)$ & $50(25.9 \%)$ & \\
\hline Moderate & $10(7.2 \%)$ & $56(29.0 \%)$ & \\
\hline Severe & $2(1.4 \%)$ & $30(15.5 \%)$ & \\
\hline \multicolumn{4}{|l|}{ Urinary pain } \\
\hline No & $123(88.5 \%)$ & $142(74.0 \%)$ & \multirow[t]{4}{*}{0.011} \\
\hline Mild & $11(7.9 \%)$ & $32(16.7 \%)$ & \\
\hline Moderate & $5(3.6 \%)$ & $16(8.3 \%)$ & \\
\hline Severe & $0(0.0 \%)$ & $2(1.0 \%)$ & \\
\hline Medical treatment after surgery & $130(60.9 \%)$ & $154(78.2 \%)$ & 0.3904 \\
\hline
\end{tabular}

Values are mean $+\mathrm{SD}$ or $n(\%)$

$B M I$ body mass index, OMA ovarian endometriosis, DIE deep infiltrating endometriosis, SUP superficial endometriosis
$19.9 \%, p<0.0001)$, and mental health disorders $(35.3 \%$ vs $3.7 \%, p<0.0001$ ) (Table 2). Among the comorbid autoimmune diseases, the Italian population showed more thyroiditis (17.9\% vs $3.4 \%$ ), dermatological diseases (psoriasis and pemphigus) (4.1\% vs 0 ) and neuromyopathies (multiple sclerosis and myasthenia gravis) (2.6\% vs 0$)$ than the Chinese population. Similarly, some of the comorbid metabolic/ endocrine diseases were more common in Italian patients 
Table 2 Gynecological and systemic comorbidities of endometriosis in the two populations

\begin{tabular}{llll}
\hline & Chinese $(n=175)$ & Italian $(n=196)$ & $p$ value \\
\hline Gynecological comorbidities & & & \\
No & $104(68.9 \%)$ & $115(60.5 \%)$ & $<0.0001$ \\
PCOS & $12(7.9 \%)$ & $10(5.3 \%)$ & \\
Adenomyosis & $15(9.9 \%)$ & $53(27.9 \%)$ & \\
Uterine fibroids & $14(9.3 \%)$ & $6(3.2 \%)$ & \\
Multiple & $6(4.0 \%)$ & $6(3.2 \%)$ & $<0.0001$ \\
Before endometriosis diagnosis & $43 / 65(91.5 \%)$ & $3 / 25(4.0 \%)$ & $<0.0001$ \\
Autoimmune diseases & $8(4.6 \%)$ & $51(26.0 \%)$ & 0.007 \\
Before endometriosis diagnosis & $1 / 7(14.3 \%)$ & $36 / 51(70.6 \%)$ & 0.007 \\
Metabolic/endocrine diseases & $28(8.7 \%)$ & $39(14.8 \%)$ & 0.023 \\
Before endometriosis diagnosis & $2 / 13(15.4 \%)$ & $14 / 26(53.8 \%)$ & $<0.0001$ \\
Inflammatory diseases & $46(19.9 \%)$ & $95(44.5 \%)$ & 0.027 \\
Before endometriosis diagnosis & $10 / 16(62.5 \%)$ & $68 / 78(87.2 \%)$ & $<0.0001$ \\
Mental health disorders & $5(3.7 \%)$ & $77(35.3 \%)$ & 0.044 \\
Before endometriosis diagnosis & $1 / 5(20.0 \%)$ & $45 / 65(69.2 \%)$ & \\
\hline
\end{tabular}

Values are mean $+\mathrm{SD}$ or $n(\%)$.

$P C O S$ polycystic ovary syndrome. including hypertension ( $7.7 \%$ vs $1.7 \%$ ) and obesity ( $3.6 \%$ vs 0 ), as well as some inflammatory diseases, i.e., allergic asthma $(1.5 \%$ vs 0$)$ and intestinal inflammatory diseases ( $3.6 \%$ vs 0$)$. Also mental health disorders were more common in Italian patients, i.e., depression ( $19.4 \%$ vs $1.1 \%)$, anxiety ( $10.7 \%$ vs $1.7 \%$ ), and anorexia ( $1.5 \%$ vs 0 ). Whether the comorbidities were identified before or after the diagnosis of endometriosis was also evaluated, and Italian women have already been diagnosed with a systemic disease before endometriosis identification (Table 2).

No differences were found in terms of medical treatment after surgery between the two groups $(60.2 \%$ vs $78.2 \%, p=0.3904)$. Regarding pain symptoms at the follow-up visit, Italian patients suffered more frequently from severe pain than the Chinese, in terms of dysmenorrhea $(p<0.0001)$, dyspareunia $(p<0.0001)$, non-menstrual pelvic pain $(p<0.0001)$, and dysuria $(p=0.011)$ (Table 1). Accordingly, the evaluation of $\mathrm{QoL}$ in the two groups showed that the Chinese patients had both for SF-12 physical score $(46.5 \pm 8.7$ vs $41.5 \pm 9.9, p<0.0001)$ and SF-12 mental score $(47.1 \pm 8.8$ vs $37.2 \pm 10.7, p<0.0001)$ higher than the Italian patients (Fig. 1). Stratifying patients according to endometriosis phenotypes, those with DIE among Italians have significantly worse mental scores compared to Chinese ( $36.8 \pm 10.4$ vs $49.8 \pm 8.2, p=0.010$ ), whereas physical scores were not significantly different $(41.9 \pm 7.3$ vs $44.6 \pm 13.1, p=0.649)$, although the sample size in Chinese population is very small. Regarding women with only ovarian localization of endometriosis among Italians and Chinese, we confirmed that Italians reported more severe pain symptoms (dysmenorrhea $26.7 \%$ vs $10.5 \%$; dyspareunia $13.5 \%$ vs $1 \%$; non-menstrual pelvic pain $16.5 \%$ vs $0 \%$ ) and a worse QoL (SF-12 physical score $41.3 \pm 11.8$ vs 46.7 $\pm 8.5, p=0.010$; SF-12 mental score $38.5 \pm 10.9$ vs $47.4 \pm 9.0$ ).

\section{Discussion}

The present cross-sectional and multicentric study explored for the first time the difference in endometriosis phenotypes, comorbidities, and QoL between Italian and Chinese patients with history of a single surgery for endometriosis. The Italian endometriotic patients showed more frequently DIE and mixed phenotypes and systemic and gynecological comorbidities than the Chinese patients. In addition, the Italian group presented at the follow up visit within 2 years after surgery with lower SF-12 physical and mental scores and more severe pain symptoms after stratifying for endometriosis phenotype and despite no differences in terms of post-surgery hormonal medical treatment.

Generally, endometriosis has been considered as a disease of Caucasian and middle-class women who delayed childrenbearing and, at the beginning, the majority of the studies were performed in Caucasian population. In the last years, a number of studies have been published on the Chinese population and differences in genetic mutations that predispose Asian and Western populations to endometriosis have been found [14]. Furthermore, genome wide association studies reported that among North Chineses polymorphisms in rs 12700667 located within the intergenic region of $7 \mathrm{p} 15$ are associated especially with an elevated risk of OMA, which is consistent with the higher incidence of OMA found in our study [15]. On the contrary, the Italian group showed more frequently DIE and mixed phenotypes, which is consistent with worse pain symptoms and lower QoL scores [16, 17]. We may hypothesize that this finding may be due to a more accurate pre-operative imaging diagnosis, which contributes to the laparoscopic identification of deep lesions and to the adequate site-mapping of 

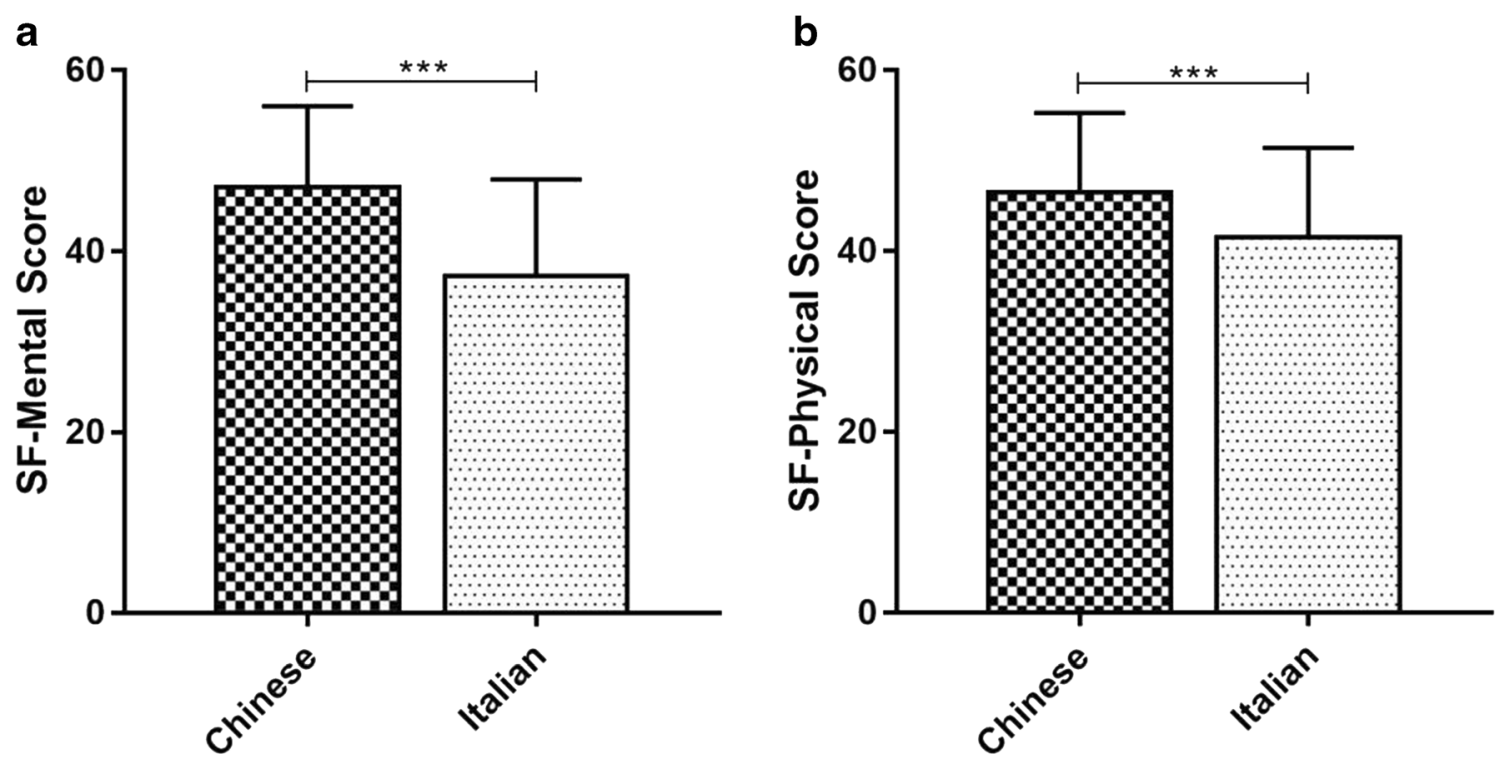

Fig. 1 The histograms showed the SF-12 physical and mental scores in Chinese and Italian population, respectively. $* * * p<0.001$

endometriosis [18]. A number of Italian gynecologists devoted to endometriosis management are themselves experts in imaging for endometriosis, which improves the preoperative diagnosis, especially of DIE lesions [19]. This difference may account for an underestimation of DIE in Chinese. However, the higher incidence of OMA among Chinese is in line with previous reports [15], and it is also consistent with their better QoL, given the lower association of this phenotype with pain symptoms, if DIE is not associated [20]. Conversely, the DIE phenotype is more frequently linked to a more severe and aggressive clinical presentation, due to specific pathogenic mechanisms such as a highly decreased apoptosis, an increased proliferation activity related to oxidative stress, a higher expression of metalloproteinases and activins for invasiveness and a relevant activation of neuroangiogenesis in ectopic endometrial lesions, compared to other phenotypes [21].

Furthermore, the two groups have significantly different baseline characteristics, such as younger age at diagnosis and more comorbidities in Italian women. No differences were found in terms of indications for surgery, with a similar rate of pain symptoms or imaging findings (i.e., adnexal mass) among the two groups. In addition, the study involved only referral hospitals for endometriosis with expertise in both surgical and medical treatment of the disease. Their protocols for endometriosis management were similar, following international guidelines $[14,22,23]$ : depending on age, pregnancy desire, pain symptoms, endometriosis phenotype, and each woman's wishes, an individualized management was planned. Younger age at surgery may denote, on one hand, a more aggressive presentation of the disease, whereas on the other it may contribute to more severe symptoms and worse QoL in Italian women with endometriosis [2]. A more relevant impact of endometriosis in Italian than Chinese women may be also explained by the difference in the medical approach, in the access to health care, in the acceptance to medical treatment between the two populations. Our study showed that Chinese underwent less frequently to a pre-operative medical hormonal treatment, whereas the rate of post-surgical hormonal treatment was approximately the same. Given the low pain scores and the better quality of life in Chinese compared to Italian group in our study, a potential role played by the Traditional Chinese Medicine (TCM) should be considered. TCM is commonly used in China to provide pain relief control, the recurrence of endometriosis following surgery, and to improve QoL [24-27]. TCM users were also less likely to require surgical treatment for endometriosis than non-users [25] and among those operated was effective in controlling recurrences [26, 27]. However, although TCM is often used for the management of endometriosis patients, there is a lack of high quality clinical evidence supporting its effectiveness compared to other medical treatments. However, as data on the use of TCM missing in our data, its potential role in contributing to pain control and to postpone first surgical intervention should be taken into account.

In other multicenter studies it was shown that ethnicity influenced the access to health care, diagnosis and treatment for endometriosis [9], thus, as a exposure factor, ethnicity throws its effect on clinical presentation and management through social and cultural constructs rather than genetics [28]. Various components of pelvic pain are the primary symptoms of endometriosis and several studies support that 
individual conceptualization of pain may be affected by different social cultures in different races [28-30]. Asian patients tend to normalize pain, while Caucasian patients more likely to seek health care positively [31]. A cross-country study reported that the incidence and the intensity of pain symptoms were significantly lower in Chinese population than Russia and France [32]. In our study, Italian patients with endometriosis suffered more from severe pelvic pain than Chinese women, suggesting a different cultural background rather than actual pain conceptualization. Also the different health system organization, with less coverage for outpatient clinic and primary care in China determining a less request for medical care, entails a diverse approach to such a chronic and multifaceted disease.

Furthermore, Italian women presented more commonly with systemic comorbidities, especially inflammatory diseases and mental health disorders (such as depression and anxiety). On the contrary, in the Chinese population, the most common comorbidities were gynecological (PCOS and uterine fibroids). Despite the prevalence of comorbidities resulted significantly different between the two groups, the diagnostic criteria of each disorders were well defined according to the current and updated guidelines, which stands for all over the world. Furthermore, the systemic comorbidity reported by the patient during medical history collection was always confirmed by reviewing medical records. Regarding the potential differences between Italy and China overall in terms of incidence of comorbidities, according to the current relevant literature there are no significant differences between the two population in terms of baseline incidence of autoimmune, inflammatory, and metabolic diseases [33, 34]. Besides, data on comorbidities among Italians are consistent with figures reported in already published studies on endometriosis [5,35-38]. The different distribution of comorbidities with endometriosis in the two population suggested a different spectrum of diseases under different society and culture. A better identification of systemic comorbidities should be taken into account in Italian group, because of the presence of a multidisciplinary team-including an immunologist, a gastroenterologist, a psychiatrist, a dietician, a neurologist, a pain specialist and a physical therapist-in the Italian Endometriosis centers. However, figures from Italians are absolutely in line with those reported in studies on cohorts from all over the world on women with endometriosis [5, 35-38]. A better investigation on systemic comorbidities among Chinese may be desirable in order to identify potential diseases accompanying endometriosis, in order to better explain clinical presentation and to plan a fully comprehensive treatment [39]. This would also allow to increase the awareness of endometriosis among specialists of other disciplines. Moreover, these comorbidities were mostly diagnosed before endometriosis, suggesting a complex inner correlation between a number of systemic diseases and predisposition to endometriosis development [5]. However, given the cross-sectional study design and a short time period of observation since the first operation for endometriosis, it is not possible to account for potential comorbidities would have developed later.

SF-12 is a validated instrument for quantifying the HealthRelated Quality Of Life (HRQOL) and higher scores on the SF-12 physical component summary (PCS) and mental component summary (MCS) indicate better quality of life. The SF12 scores of PCS and MCS were significantly higher in Chinese than in Italian patients with endometriosis, suggesting that the QoL in the Chinese patients resulted better than in the Italian patients and this does not depend on the phenotype of endometriosis. In fact, after stratifying for DIE and OMA phenotype, data on pain symptoms intensity and QoL among Chinese women were still better than in the Italian Group. As already mentioned, a potential role of TCM should be considered, even though no data are available on that variable. Probably, the better scores among Chinese patients are in part related to the lower number of comorbidities in the Chinese women, suggesting a possible poor attention to this kind of questioning when clinical history was collected. A study reported that QoL was independently associated with more severe dysmenorrhea and more severe chronic pelvic pain, but not with higher ASRM stage [40, 41]. A correlation between different phenotypes of endometriosis and levels of stress perception was observed $[1,42]$, suggesting a possible association between the forms of endometriosis and impact on QoL. Besides, the presence of psychiatric diseases was related to endometriosis-associated pain but not with lesions localization $[43,44]$. It is clear that the adverse effect of endometriosis on QoL are related to various factors, including pain/ discomfort symptoms, infertility, and high intensity of stress perception $[1,45,46]$. In addition, ethnicity may affect quality of life of endometriotic patients through different pain conceptualization and access and acceptance to health care.

Some limitations and strengths of the study should be acknowledged. The study included only women with endometriosis who underwent first surgery in referral centers for endometriosis. Currently, a large number of patients with endometriosis are only medically treated with good results and the first-line approach for endometriosis symptoms should be medical treatment [47]. However, the study is focused only on those with a previous surgical operation for endometriosis, so our results cannot be applied to all endometriosis-affected women. The choice of including only women with histological diagnosis of endometriosis aimed to have strict criteria of inclusion for the centers involved in the research, in order to minimize bias due to different imaging techniques and no universally shared diagnostic criteria for the non-invasive diagnosis of each phenotype of endometriosis. Women with history of multiple surgery were excluded, in order to reduce the impairment of QoL due to repeated surgery, relapses, and recurrences. In addition, as the study is cross-sectional it is not possible to predict which comorbidities would have developed as time passes. 
Based on the results, in young women with comorbid diseases and mild symptoms, the possibility of endometriosis should be considered in order to not delay the diagnosis of endometriosis. Moreover, more and more patients with endometriosis-associated pain required a better treatment of the various comorbidities in order to improve their quality of life.

Author Contributions $\mathrm{HC}, \mathrm{TC}, \mathrm{YW}, \mathrm{SV}$, and $\mathrm{HS}$ collected the data in each center. SV analyzed the database and wrote the first draft with HC. $\mathrm{MC}, \mathrm{LM}, \mathrm{YW}, \mathrm{HH}$, and FP interpreted the results and finally revised the manuscript for important intellectual contribution.

Funding Open access funding provided by Università degli Studi di Firenze within the CRUI-CARE Agreement.

\section{Declarations}

Conflicts of Interest The author reported no conflict of interest.

Open Access This article is licensed under a Creative Commons Attribution 4.0 International License, which permits use, sharing, adaptation, distribution and reproduction in any medium or format, as long as you give appropriate credit to the original author(s) and the source, provide a link to the Creative Commons licence, and indicate if changes were made. The images or other third party material in this article are included in the article's Creative Commons licence, unless indicated otherwise in a credit line to the material. If material is not included in the article's Creative Commons licence and your intended use is not permitted by statutory regulation or exceeds the permitted use, you will need to obtain permission directly from the copyright holder. To view a copy of this licence, visit http://creativecommons.org/licenses/by/4.0/.

\section{References}

1. Lazzeri L, Orlandini C, Vannuccini S, Pinzauti S, Tosti C, Zupi E, et al. Endometriosis and perceived stress: impact of surgical and medical treatment. Gynecol Obstet Investig. 2015;79:229-33.

2. Vannuccini S, Reis FM, Coutinho LM, Lazzeri L, Centini G, Petraglia F. Surgical treatment of endometriosis: prognostic factors for better quality of life. Gynecol Endocrinol. 2019;35:1010-4.

3. Guo SW. Recurrence of endometriosis and its control. Hum Reprod Update. 2009;15:441-61.

4. Capezzuoli T, Vannuccini S, Fantappie G, Orlandi G, Rizzello F, Coccia ME, et al. Ultrasound findings in infertile women with endometriosis: evidence of concomitant uterine disorders. Gynecol Endocrinol. 2020;36:808-812.

5. Kvaskoff M, Mu F, Terry KL, Harris HR, Poole EM, Farland L, et al. Endometriosis: a high-risk population for major chronic diseases? Hum Reprod Update. 2015;21:500-16.

6. Vannuccini S, Lazzeri L, Orlandini C, Morgante G, Bifulco G, Fagiolini A, et al. Mental health, pain symptoms and systemic comorbidities in women with endometriosis: a cross-sectional study. J Psychosom Obstet Gynaecol. 2018;39:315-20.

7. Surrey ES, Soliman AM, Johnson SJ, Davis M, Castelli-Haley J, Snabes MC. Risk of developing comorbidities among women with endometriosis: a retrospective matched cohort study. J Women's Health. 2018;27:1114-23.
8. Bougie O, Healey J, Singh SS. Behind the times: revisiting endometriosis and race. Am J Obstet Gynecol. 2019;221:35.e1-5.

9. Bougie O, Yap MI, Sikora L, Flaxman T. Influence of race/ ethnicity on prevalence and presentation of endometriosis: a systematic review and meta-analysis. BJOG. 2019;126:1104-15.

10. Fawole AO, Bello FA, Ogunbode O, Odukogbe AT, Nkwocha GC, Nnoaham KE, et al. Endometriosis and associated symptoms among Nigerian women. Int J Gynaecol Obstet. 2015;130:190-4.

11. Flores I, Abreu S, Abac S, Fourquet J, Laboy J, Ríos-Bedoya C. Self-reported prevalence of endometriosis and its symptoms among Puerto Rican women. Int J Gynaecol Obstet. 2008;100:257-61.

12. Yen CF, Kim MR, Lee CL. Epidemiologic factors associated with endometriosis in East Asia. Gynecol Minim Invasive Ther. 2019;8: 4-11.

13. Gandek B, Ware JE, Aaronson NK, Apolone G, Bjorner JB, Brazier JE, et al. Cross-validation of item selection and scoring for the SF12 Health Survey in nine countries: results from the IQOLA project. International quality of life assessment. J Clin Epidemiol. 1998;51: 1171-8.

14. Dai Y, Li X, Shi J, Leng J. A review of the risk factors, genetics and treatment of endometriosis in Chinese women: a comparative update. Reprod Health. 2018;15:82.

15. Li Y, Hao N, Wang Y, Kang S. Association of endometriosisassociated genetic polymorphisms from genome-wide association studies with ovarian endometriosis in a Chinese population. Reprod Sci. 2017;24:109-13.

16. Koninckx PR, Ussia A, Adamyan L, Wattiez A, Donnez J. Deep endometriosis: definition, diagnosis, and treatment. Fertil Steril. 2012;98:564-71.

17. Montanari E, Dauser B, Keckstein J, Kirchner E, Nemeth Z, Hudelist G. Association between disease extent and pain symptoms in patients with deep infiltrating endometriosis. Reprod BioMed Online. 2019v:39:845-51.

18. Exacoustos C, Malzoni M, Di Giovanni A, Lazzeri L, Tosti C, Petraglia F, et al. Ultrasound mapping system for the surgical management of deep infiltrating endometriosis. Fertil Steril. 2014;102: 143-150.e2.

19. Exacoustos C, Lazzeri L, Zupi E. Expert sonographers and surgeons are needed to manage deep infiltrating endometriosis. Ultrasound Obstet Gynecol. 2017;49:417.

20. Chapron C, Santulli P, de Ziegler D, Noel JC, Anaf V, Streuli I, et al. Ovarian endometrioma: severe pelvic pain is associated with deeply infiltrating endometriosis. Hum Reprod. 2012;27:702-11.

21. Tosti C, Pinzauti S, Santulli P, Chapron C, Petraglia F. Pathogenetic mechanisms of deep infiltrating endometriosis. Reprod Sci. 2015;22:1053-9.

22. Dunselman GAJ, Vermeulen N, Becker C, Calhaz-Jorge C, D'Hooghe T, De Bie B, et al. ESHRE guideline: management of women with endometriosis. Hum Reprod. 2014;29:400-12.

23. ETIC Endometriosis Treatment Italian Club. When more is not better: 10 'don'ts' in endometriosis management. An ETIC (*) position statement. Hum Reprod Open. 2019;2019:hoz009.

24. Zhu S, Liu D, Huang W, Wang Q, Wang Q, Zhou L, et al. Postlaparoscopic oral contraceptive combined with Chinese herbal mixture in treatment of infertility and pain associated with minimal or mild endometriosis: a randomized controlled trial. BMC Complement Altern Med. 2014;14:222.

25. Su SY, Muo CH, Sung FC, Morisky DE. Reduction of surgery rate in endometriosis patients who take Chinese medicine: a populationbased retrospective cohort study. Complement Ther Med. 2014;22: $632-9$.

26. Zhao R, Hao Z, Zhang Y, Lian F, Sun W, Liu Y, et al. Controlling the recurrence of pelvic endometriosis after a conservative operation: comparison between Chinese herbal medicine and western medicine. Chin J Integr Med. 2013;19:820-5. 
27. Zhao R, Liu Y, Tan Y, Hao Z, Meng Q, Wang R, et al. Chinese medicine improves postoperative quality of life in endometriosis patients: a randomized controlled trial. Chin J Integr Med. 2013;19:15-21.

28. Yudell M, Roberts D, DeSalle R, Tishkoff S. SCIENCE AND SOCIETY. Taking race out of human genetics. Science. 2016;351(6273):564-5.

29. Kwok W, Bhuvanakrishna T. The relationship between ethnicity and the pain experience of cancer patients: a systematic review. Indian J Palliat Care. 2014;20:194-200.

30. Frisch S. Perceptions of pain. Cultural differences add to the challenge of treating patients' pain. Minn Med. 2014;97:14-6.

31. Campbell CM, Edwards RR. Ethnic differences in pain and pain management. Pain Manag. 2012;2:219-30.

32. Chapron C, Lang JH, Leng JH, Zhou Y, Zhang X, Xue M, et al. Factors and regional differences associated with endometriosis: a multi-country, case-control study. Adv Ther. 2016;33(8):1385407.

33. Li R, Sun X, Liu X, Yang Y, Li Z. Autoimmune diseases in China. Adv Immunol. 2019;144:173-216.

34. Ng SC, Tang W, Ching JY, Wong M, Asia-Pacific Crohn's and Colitis Epidemiologic Study (ACCESS) Study Group, et al. Incidence and phenotype of inflammatory bowel disease based on results from the Asia-pacific Crohn's and colitis epidemiology study. Gastroenterology. 2013;145:158-165.e2.

35. Shigesi N, Kvaskoff M, Kirtley S, Feng Q, Fang H, Knight JC, et al. The association between endometriosis and autoimmune diseases: a systematic review and meta-analysis. Hum Reprod Update. 2019;25:486-503.

36. Chiaffarino F, Cipriani S, Ricci E, Roncella E, Mauri PA, Parazzini $\mathrm{F}$, et al. Endometriosis and inflammatory bowel disease: a systematic review of the literature. Eur J Obstet Gynecol Reprod Biol. 2020;252:246-51.

37. Parazzini F, Esposito G, Tozzi L, Noli S, Bianchi S. Epidemiology of endometriosis and its comorbidities. Eur J Obstet Gynecol Reprod Biol. 2017;209:3-7.
38. Bungum HF, Vestergaard C, Knudsen UB. Endometriosis and type 1 allergies/immediate type hypersensitivity: a systematic review. Eur J Obstet Gynecol Reprod Biol. 2014;179:209-15.

39. Teng SW, Horng HC, Ho CH, Yen MS, Chao HT, Wang PH, et al. Women with endometriosis have higher comorbidities: analysis of domestic data in Taiwan. J Chin Med Assoc. 2016;79:577-82.

40. McPeak AE, Allaire C, Williams C, Albert A, Lisonkova S, Yong PJ. Pain Catastrophizing and pain health-related quality-of-life in endometriosis. Clin J Pain. 2018;34:349-56.

41. Facchin F, Barbara G, Saita E, Mosconi P, Roberto A, Fedele L, et al. Impact of endometriosis on quality of life and mental health: pelvic pain makes the difference. J Psychosom Obstet Gynaecol. 2015;36:135-41.

42. Luisi S, Pizzo A, Pinzauti S, Zupi E, Centini G, Lazzeri L, et al. Neuroendocrine and stress-related aspects of endometriosis. Neuro Endocrinol Lett. 2015;36:15-23.

43. Sepulcri RP, do Amaral VF. Depressive symptoms, anxiety, and quality of life in women with pelvic endometriosis. Eur J Obstet Gynecol Reprod Biol. 2009; 142:53-6.

44. Eriksen HL, Gunnersen KF, Sorensen JA, Munk T, Nielsen T, Knudsen UB. Psychological aspects of endometriosis: differences between patients with or without pain on four psychological variables. Eur J Obstet Gynecol Reprod Biol. 2008;139:100-5.

45. Reis FM, Coutinho LM, Vannuccini S, Luisi S, Petraglia F. Is stress a cause or a consequence of endometriosis? Reprod Sci. 2020;27: $39-45$.

46. Nnoaham KE, Hummelshoj L, Webster P, d'Hooghe T, de Cicco Nardone F, de Cicco Nardone C, et al. Impact of endometriosis on quality of life and work productivity: a multicenter study across ten countries. Fertil Steril. 2011;96:366-73.e8.

47. Vercellini P, Buggio L, Frattaruolo MP, Borghi A, Dridi D, Somigliana E. Medical treatment of endometriosis-related pain. Best Pract Res Clin Obstet Gynaecol. 2018;51:68-91.

Publisher's Note Springer Nature remains neutral with regard to jurisdictional claims in published maps and institutional affiliations. 